\title{
COMMENTARY
}

\section{Weighing In On the Role of Pharmacy Education in Mitigating Weight Bias}

\author{
Amelia Winters, PharmD, ${ }^{\mathrm{a}}$ Hannah E. Johnson, PharmD ${ }^{\mathrm{b}}$ \\ ${ }^{\text {a }}$ St. Claire HealthCare, Department of Pharmacy, Morehead, Kentucky \\ ${ }^{\mathrm{b}}$ University of Kentucky, College of Pharmacy, Lexington, Kentucky
}

Corresponding Author: Hannah E. Johnson, University of Kentucky, College of Pharmacy, 789 S. Limestone Str, Lexington, KY 40536. Tel: 859-323-7499. Email: hannahejohnson@uky.edu

Submitted August 9, 2021; accepted January 3, 2022; ePublished January 2022

Two-thirds of American adults are labeled as overweight or obese by current body mass index (BMI) weight status categories. Individuals categorized as overweight or obese are at risk for weight bias and subsequently poorer health care experiences and outcomes. However, schools and colleges of pharmacy may not be providing robust education or training to student pharmacists on weight bias, assessment of their own bias, or how to address and mitigate weight bias against patients. This commentary explores the impact of weight bias on patient care and how efforts can be advanced to recognize and address weight bias in pharmacy education and pharmacy practice to provide optimal care to patients of all sizes.

Keywords: weight bias, stigma, pharmacy education, health outcomes, curriculum

\section{INTRODUCTION}

Pharmacists provide education and pharmacological and non-pharmacological recommendations for treatment and prevention of illness and health promotion. One area pharmacists may find themselves offering guidance and making recommendations for is weight management. This can occur through weight management programs, in the setting of treatment of concurrent health conditions, or with individuals with no known health problems. ${ }^{1}$ No matter the setting, pharmacists remain accessible health care professionals who improve the health of patients, reduce suffering and advocate for patients and changes that improve patient care. Issues of bias are an unfortunate reality for patients. One form of bias that has not been reviewed or extensively documented in pharmacy education or practice is weight bias. ${ }^{1}$ Approximately two-thirds of adults in the United States are considered to be overweight or obese as calculated by body mass index $(\mathrm{BMI}){ }^{2}$ These individuals are at risk for the negative impacts of weight bias, including stigma and discrimination, which can put an individual at risk for low self-esteem, depression, and lower quality of life. ${ }^{3}$ There is an integral role for pharmacists to advocate to improve the well-being of the vast majority of our patients and reduce their vulnerability to psychological and social distress. ${ }^{4}$

Weight bias is defined as the negative attitudes and beliefs towards others due to their weight. This form of bias can affect anyone, but most commonly people who are labeled overweight or obese. People can experience this bias in any aspect of life, including at the workplace, in health care, among interpersonal relationships, and within education. ${ }^{4}$ Weight bias can manifest as stigma, stereotypes, prejudice, and discrimination. Weight stigma, or marking an individual as having a lower social value because of their weight, can lead to discrimination. ${ }^{3}$ In employment settings and daily interpersonal relationships, weight discrimination has been reported as often as race discrimination, and in some adults, more often than gender or age discrimination. ${ }^{5}$ Weight discrimination may be most prevalent in minority ethnic and racial groups. ${ }^{5}$

The latest Center for the Advancement of Pharmacy Education (CAPE) 2013 Educational Outcomes include patient advocacy and cultural sensitivity as part of the structured framework for pharmacy educational outcomes, noting that students should "assure that patients' best interests are represented" and "recognize social determinants of health to diminish disparities and inequities in access to quality care", respectively. ${ }^{6}$ However, there is limited guidance on how best to implement this within pharmacy curriculum. Education on the multifactorial and non-uniform influences on obesity is necessary for the profession to understand patients' experiences and behaviors, to mitigate weight bias, and thus improve patient care. ${ }^{7,8}$

\section{DISCUSSION}

Health care Disparities and the Impact of Weight Bias 
Weight bias negatively impacts health. Regardless of BMI, it has been found that people who perceive themselves as heavy experience the stress of weight bias and have corresponding elevated cortisol, which is linked to negative health outcomes, stimulates eating, and directly promotes abdominal adiposity, therefore continuing to perpetuate weight bias. ${ }^{9}$ People experiencing weight bias are also at increased risk of psychological issues such as depression, anxiety, disordered eating, and body dissatisfaction. ${ }^{3,4,9}$ A stronger association has been found between weight discrimination and mortality rates compared to other attributions for discrimination, such as race, ethnicity, sex, age, appearance, or sexual orientation. $^{10}$

Because weight bias is present in all aspects of society and that the resulting discrimination is harmful to the health of its victims, it is important to first review how pervasive weight bias is in health care. High levels of weight bias have been found among health care clinicians, including primary care providers and health promotion specialists, resulting in beliefs that larger patients are lazy, weak-willed, and bad. Those same clinicians report having less respect for largerbodied patients and see them as "a waste of time". These beliefs were found to lead to lower quality of care. ${ }^{3}$ Physicians also engage in less health education with higher weight patients and $69 \%$ of respondents in a survey reported that doctors were the source of their experiences with weight bias. ${ }^{11,12}$ These biases and resulting behaviors are present in other health care professions, including nurses and psychologists. Due to poor experiences with health care providers or expectations for poor treatment, larger patients may avoid seeking medical attention, mistrust providers, and have higher risk for treatment non-adherence.

\section{Recognizing Weight Bias}

The first step in addressing weight bias is to identify and recognize one's own biases. Bias is characterized as either implicit or explicit. Implicit biases are automatic, often unconscious, and impact one's behavior without awareness. Implicit biases are commonly assessed through latency-response tests, such as the Implicit Association Test (IAT) from Project Implicit, which measures the strength of associations between concepts and evaluations of stereotypes. ${ }^{13}$ The Thin-Fat IAT utilizes pictures of fat and thin people and compares time required to categorize these pictures with positive (ex. beautiful, joyful) and negative (ex. ugly, awful) words. ${ }^{14,15}$ Explicit biases are conscious, intentional, and assessed through self-reporting tools. Explicit bias tools for weight stigma include the Crandall's anti-fat attitudes questionnaire (AFAT) or asking feelings or preferences towards thin versus larger-bodied people, such as:

What stereotypes do I have about people with obesity? How do I feel when I work with patients of different body sizes? Do I make assumptions regarding a person's character, intelligence, abilities, health status or behaviors based only on their weight? How do my patients in larger bodies feel when they leave? Do they feel confident and empowered, or otherwise? ${ }^{12,13,15}$

With this understanding of weight bias and its negative effects in health care, health professional schools and colleges have sought to determine the prevalence of weight bias amongst its students. In a large national sample of medical students, the majority exhibited weight bias comparable to or with scores more negative towards people identified as obese than toward racial and ethnic minority groups. ${ }^{13}$ Implicit bias towards overweight individuals was also identified in a study of nursing and psychology students. ${ }^{16}$ Limited information exists on weight bias in pharmacists or student pharmacists. The profession has been minimally assessed alone or grouped with other health care professionals in studies that have shown both implicit and explicit weight bias. ${ }^{1,17}$ Clements and Akins's recent study of 84 student pharmacists at one college of pharmacy found neutral attitudes towards obesity. However, only measures for explicit bias were used. ${ }^{18}$

\section{Addressing Weight Bias}

There is limited information on how weight bias is being addressed within schools or colleges of pharmacy. ${ }^{1,18}$ At the University of Kentucky College of Pharmacy, weight bias is not mapped in the curriculum and was found to only be listed as a form of bias in an unconscious bias training course. Curriculum mapping and a better understanding of the current extent in which weight bias is taught in schools and colleges of pharmacy will provide additional insight into the additional need for education and assurance that CAPE outcomes are met. Expansion of the entrustable professional activities (EPAs) for new pharmacy graduates to include sensitivity and communication skills with diverse populations should also be considered. ${ }^{19}$

Within the discussions of advocacy, sensitivity, and health disparities in pharmacy curricula, weight bias should be included. This can occur concomitantly with discussions of other forms of bias or throughout the curriculum, as the topic of weight or obesity show up, such as in courses focused on wellness and health promotion, nutrition, endocrine disorders, cardiology, public health and policy, and patient care lab. Education on the complex genetic and environmental influences should occur concurrently. ${ }^{7}$ Specific recognition of and concurrent education about the uncontrollable and nonmodifiable causes of obesity, including genetics and biology, as well as the complex social determinants and 
environmental factors which may impact behaviors or obesity, such as sociodemographic, socioeconomic, and physical environments should be included. ${ }^{7,8}$ Although BMI is most often used to define obesity, it is not a direct measure of body fat or health. Therefore, alternative definitions and assessments of obesity and health should be described, such as alternative measurement tools, physical activity and dietary intake. ${ }^{20}$ Inclusion and input from people with lived experiences should be utilized when discussing weight bias. ${ }^{7}$ This may include guest panel discussions and didactic lectures or inclusion of these topics or exposure in already existing standardized patient scenarios, case-based learning exercises, and implicit bias or cultural competency training. Additionally, schools or colleges of pharmacy should ensure a body-inclusive environment, including furniture, equipment (ex. blood pressure cuffs), and space (ex. distance between furniture) for students, faculty, and guests of all sizes to feel comfortable.

Sensitivity training, specifically including weight bias, should be provided to all faculty, staff, and students. Educating future pharmacists on weight bias requires faculty to take time to assess their own implicit and explicit biases using tools like the Thin-FAT IAT and AFAT, respectively, and critically reflect on how this may impact not just what they teach, but how they teach. Faculty should pay close attention to not propagate weight bias in and out of the classroom and avoid the use of stigmatizing and blaming words. Person-first language should be used in lectures, active-learning sessions, and introductory and advanced pharmacy practice experiences with emphasis on the person and not a characteristic or diagnosis. For example, use "person with obesity" or "patient affected by obesity", instead of "obese patient" or "morbidly obese". ${ }^{7,12}$ Faculty should facilitate a safe space for learners and patients and not accept or tolerate weight bias in any form, serving as a mentor and modeling advocacy. If miscommunication occurs, recognition of the mistake and apologies should be provided.

Evaluation and assessment of weight biases can be completed by students during active-learning sessions, interprofessional education (IPE) or self-reflection to gain understanding of their own personal biases. IPE provides an opportunity for student pharmacists to work with other health professions students to collaboratively treat patients with a weight-neutral and inclusive approach. It can also be used to develop and find resources, such as health care providers who provide weight-inclusive care. Students should practice speaking up against biases, such as weight bias and have the tools to advocate for patients if they are discriminated against.

\section{CONCLUSION}

Pharmacists and future pharmacists are in an optimal position to mitigate weight bias and discrimination in many areas, including pharmacy curriculum, retail, hospital and ambulatory settings which will help improve the health care experience and increase access for millions of people.

\section{REFERENCES}

1. Murphy AL, Gardner DM. A scoping review of weight bias by community pharmacists towards people with obesity and mental illness. Can Pharm J. 2016 Jul;149(4):226-35.

2. Fryar CD, Carroll MD, Afful J. Prevalence of overweight, obesity, and severe obesity among adults aged 20 and over: United States, 1960-1962 through 2017-2018. NCHS Health E-Stats; 2020. Accessed July 28, 2021.

https://www.cdc.gov/nchs/data/hestat/obesity-adult-17-18/obesity-adult.htm

3. Phelan SM, Burgess DJ, Yeazel MW, et al. Impact of weight bias and stigma on quality of care and outcomes for patients with obesity. Obes Rev. 2015;16(4):319-326.

4. Puhl RM, Heur CA. The stigma of obesity: a review and update. Obesity. 2009;17:941-964.

5. Puhl RM, Andreyeva T, Brownell KD. Perceptions of weight discrimination: prevalence and comparison to race and gender discrimination in America. Int J Obes. 2008;32(6):992-1000.

6. Medina MS, Plaza CM, Stowe CD, et al. Center for the Advancement of Pharmacy Education 2013 educational outcomes. Am J Pharm Educ. 2013;77(8):162.

7. Ramos Salas X, Alberga AS, Cameron E, Estey L, Forhan M, Kirk SFL, Russell-Mayhew S, Sharma AM. Addressing weight bias and discrimination: moving beyond raising awareness to creating change. Obes Rev. 2017;18(11):13231335.

8. Akram H, Ashraf G, Ijaz MA. The impacts of complex social, environmental, and behavioral factors of obesity. Int $J$ Basic Sci Med. 2018;3(3):94-98.

9. Himmelstein MS, Incollingo Belsky AC, Tomiyama AJ. The weight of stigma: Cortisol reactivity to manipulated weight stigma. Obesity. 2014;23(2):368-374.

10. Sutin AR, Stephan Y, Terracciano A. Weight discrimination and risk of mortality. Psychol Sci. 2015;26(11):18031811.

11. Bertakis KD, Azari R. The impact of obesity on primary care visits. Obes Res. 2005;13(9):1615-1623. 
12. The Obesity Action Coalition. Weight Bias in Healthcare: A Guide for Healthcare Providers Working with Individuals Affected by Obesity; 2015. Accessed July 1, 2021. https://www.obesityaction.org/action-throughadvocacy/weight-bias/weight-bias-guides/.

13. Phelan SM, Dovidio JF, Puhl RM, et al. Implicit and explicit weight bias in a national sample of 4,732 medical students: the medical student CHANGES study. Obesity (Silver Spring). 2014 Apr;22(4):1201-8.

14. Greenwald AG, et al. Understanding and using the Implicit Association Test: III. Meta-analysis of predictive validity. J Pers Soc Psychol. 2009; 97(1):17-41.

15. Elran-Barak R, Bar-Anan Y. Implicit and explicit anti-fat bias: The role of weight-related attitudes and beliefs. Soc Sci Med. 2018;204:117-124.

16. Waller T, Lampman C, Lupfer-Johnson G. Assessing bias against overweight individuals among nursing and psychology students: an implicit association test. J Clin Nurs. 2012;21:3504-3512.

17. Teachman BA, Brownell KD. Implicit anti-fat bias among health professionals: is anyone immune? Int J Obes Relat Metab Disord. 2001;25(10):1525-1531.

18. Clements JN, Akins KB. A cross-sectional study of student pharmacists' attitudes and self-awareness towards obesity and weight. Curr Pharm Teach Learn. 2021;13(12):1654-1658.

19. Haines ST, Pittenger AL, Stolte SK, et al. Core Entrustable Professional Activities for New Pharmacy Graduates. Am J Pharm Educ. 2017;81(1):S2.

20. Jensen MD, Ryan DH, Donato KA, et al. Executive summary: Guidelines (2013) for the management of overweight and obesity in adults. Obesity. 2014;22:S5-S39. 